\title{
Iron line signatures in X-ray afterglows of GRB by BeppoSAX
}

\author{
L. Piro ${ }^{1}$, E. Costa ${ }^{1}$, M. Feroci ${ }^{1}$, G. Stratta ${ }^{1}$, F. Frontera ${ }^{2,3}$, L. Amati ${ }^{2}$, D. Dal Fiume ${ }^{2}$, L.A. Antonelli ${ }^{4,5}$, J. Heise ${ }^{6}$, \\ J. in 't Zand ${ }^{6}$, A. Owens ${ }^{7}$, A.N. Parmar ${ }^{7}$, G. Cusumano ${ }^{8}$, M. Vietri ${ }^{9}$, and G.C. Perola ${ }^{9}$ \\ 1 Istituto di Astrofisica Spaziale, C.N.R., Roma, Italy \\ 2 Istituto T.E.S.R.E., C.N.R., Bologna, Italy \\ 3 Dipartimento di Fisica, Universita' di Ferrara, Italy \\ 4 BeppoSAX Science Data Center, Rome, Italy \\ 5 Osservatorio Astronomico di Roma, Italy \\ 6 Space Research Organization in the Netherlands, Utrecht, The Netherlands \\ 7 Space Science Department of ESA, ESTEC \\ 8 Istituto Fisica Cosmica e Appl. Calc. Informatico, C.N.R., Palermo, Italy \\ 9 Dipartimento di Fisica, Universita' Roma Tre, Roma, Italy
}

Received March 16; accepted June 25, 1999

\begin{abstract}
We report the possible detection (99.3\% of statistical significance) of redshifted Fe iron line emission in the X-ray afterglow of Gamma-ray burst GRB 970508 observed by BeppoSAX. Its energy is consistent with the redshift of the putative host galaxy determined from optical spectroscopy. In contrast to the fairly clean environment expected in the merging of two neutron stars, the observed line properties would imply that the site of the burst is embedded in a large mass of material $\left(>0.5 M_{\odot}\right)$, consistent with pre-explosion ejecta of a very massive star. This material could be related with the outburst observed in the afterglow 1 day after the GRB and with the spectral variations measured during this phase. We did not find evidence of Fe line in two other GRB with known redshift (GRB 971214 and GRB 980613), but we note that the upper limits are of the same order of the intensity measured in GRB 97508 and that none of these afterglows shows rebursting activity.
\end{abstract}

Key words: gamma-ray: bursts

\section{Introduction}

Distance - scale determination of Gamma-ray bursts (GRB) has been one of the most important achievements of astrophysics in recent years. Accurate and fast localization of the prompt and afterglow emission (Piro et al. 1998a; Costa et al. 1997) by BeppoSAX (Piro et al. 1995; Boella et al. 1997) led to the identification of optical counterparts (van Paradijs et al. 1997) and ultimately to spectral measurements of a redshift (Metzger et al. 1997). While the extragalactic origin of GRB has gathered solid evidence in its support, the source of the large energy implied by their distance is still speculative.

Send offprint requests to: piro@ias.rm.cnr.it
The measurement of X-ray Fe lines emitted directly by the GRB or its afterglow could provide a direct measurement of the distance and probe into the nature of the central environment (Perna \& Loeb 1998; Mészáros \& Rees 1998; Boettcher et al. 1999; Ghisellini et al. 1998). Neutron star - neutron star merging should happen in a fairly clean environment, with line intensities much below the sensitivity of current experiments. In contrast, Mészáros \& Rees (1998) have shown that the circumburst environment created by the stellar wind before the explosion of the hypernova could yield a line of substantial intensity. A similarly favourable situation should be expected in a variation of the hypernova scenario, - the SupraNova (Vietri \& Stella 1998), where the GRB is shortly preceded by a supernova explosion with the ejection of an iron-rich massive shell. It is also conceivable that the impact of the relativistic shell that produced the original GRB on these ejecta could provide an additional energy input in the afterglow.

Motivated by these expectations, we have started a detailed analysis of afterglow spectra to look for the presence of features. The first and most promising candidate is GRB 970508. It is characterized by a large outbursting event during its afterglow phase (Piro et al. 1998b) and has the highest signal to noise ratio of the BeppoSAX GRB afterglows. Here we summarize the results obtained in this burst (reported more extensively in Piro et al. 1999) and present the first results of the analysis in other afterglows.

\section{Possible evidence of Fe line feature in GRB 970508}

We have searched the X-ray spectrum of GRB 970508's afterglow for an iron line, located at the system's redhift $(z=0.835$, Bloom et al. 1998); we found such a line with limited statistical significance $(99.3 \%)$ in the early part (first $16 h$ ) of the afterglow; the line decreases in the later part of the observations $(\approx 1$ day after the burst) 
Table 1. Iron lines in X-ray afterglows of GRB with known $z$

\begin{tabular}{llllll}
\hline GRB & $T_{\text {start }}^{1}$ & $F_{\mathrm{c}}^{2}$ & $I_{\mathrm{L}}^{3}$ & E.W. $^{4}$ & $z$ \\
970508 & 6 & 6 & $5 \pm 2$ & $3 \pm 1.4$ & 0.835 \\
971214 & 7 & 7 & $<6$ & $<0.5$ & 3.14 \\
980613 & 9 & 3 & $<2.5$ & $<2$ & 1.096 \\
\hline
\end{tabular}

Note: Continuum and line intensities in the period $T_{\text {start }}$ to $T_{\text {start }}+30 \mathrm{ksec}$; Errors and upper limits correspond to $90 \%$ confidence level for 1 parameter of interest; $z$ of GRB 971214 from Kulkarni et al. (1998); $z$ of GRB 980613 from Djorgovski et al. (1999). ${ }^{1}$ observation start in hrs from the GRB; ${ }^{2} F(2-10 \mathrm{keV})$ in $10^{-13} \mathrm{erg} \mathrm{cm}^{-2} \mathrm{~s}^{-1} ;{ }^{3}$ Line intensity in $10^{-5} \mathrm{ph} \mathrm{cm}^{-2} \mathrm{~s}^{-1}$; ${ }^{4}$ observed E.W. in keV.

by at least a factor 2, enough to make it undetectable with current apparatus (Fig. 1). Simultaneously with the line disappearance, the $\mathrm{X}$-ray flux both rises and hardens ( $\alpha=0.4 \pm 0.6$, while $\alpha=1.5 \pm 0.6$ before the reburst), consistent with the appearance of a new shock. Then, at the end of the outburst, the spectrum steepens.

\section{Search of Fe line in other GRB's afterglows}

An extensive analysis is under way to search for features in all the X-ray afterglows observed by BeppoSAX. We report here the results derived in relatively bright afterglow sources (flux in the first part of the observation $>310^{-13} \mathrm{erg} \mathrm{cm}^{-2} \mathrm{~s}^{-1}$ ) which have a spectroscopic redshift determination, thus allowing to fix the energy of the line. In the two GRB's that meet these conditions (GRB 971214 and GRB 980613) we find upper limits that are roughly consistent with the intensity measured in GRB 970508 (Table 1).

\section{Origin of the line and constraints on the emitting region}

It is most likely that the line of GRB 970508 is produced by fluorescence and recombination of $\mathrm{Fe}$ atoms ionized by the intense flux of the GRB and its afterglow (Piro et al. 1999; Lazzati et al. 1999). In the early phases of the GRB the radiation field is so high that iron atoms are completely stripped of their electrons: the Compton temperature is very high and then recombination is not very efficient in producing line photons. When the flux decreases, about $10^{4} \mathrm{~s}$ after the burst, fluorescence becomes an effective process. We note, in passing, that the intensity of the line is therefore not correlated with the luminosity of the burst: for example, with a luminosity a factor of 10 larger, the medium would have remained completely ionized upto about 1 day after the burst, producing therefore a line with a lower intensity. The minimum mass needed to produce the line is (Piro et al. 1999; Lazzati et al. 1999) $M_{\min }=0.5 M_{\odot} A_{\mathrm{Fe}}^{-1}$, where $A_{\mathrm{Fe}}$ is the iron abundance normalized to the solar value. From the line variability, intensity and width we deduce that this medium should be located at a distance of $\approx 310^{15} \mathrm{~cm}$ from the central source, it is moving with subrelativistic speed, it should have a large density $\left(n>510^{9} \mathrm{~cm}^{-3}\right)$, and it should lie sideways respect to the observer, otherways it would smear out the short timescale structure
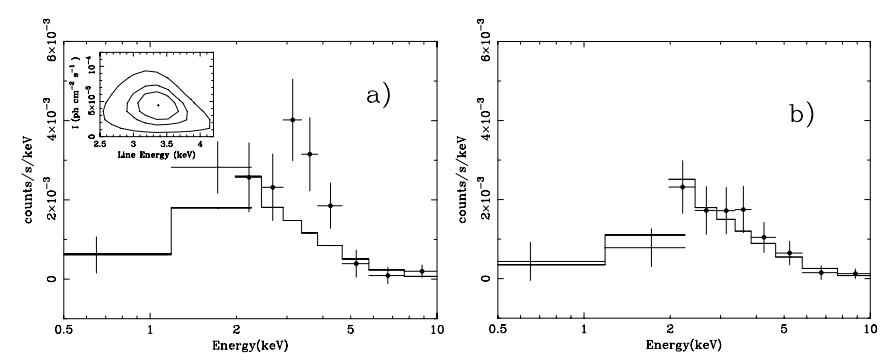

Fig. 1. The spectra (in detector counts) of the afterglow of GRB 970508 in the first a) and and second part of the observation b) fitted with a power law continuum

of the burst with Thomson scattering (Boettcher et al. 1999).

In order to reach such distance, this material must have been pre-ejected by the source originating the burst, shortly (perhaps a year, for typical SN expansion speeds) before the burst. We stress that these observations contain two coincidences: on the one hand, this is the only burst in which a reburst and a line have been observed by BeppoSAX; on the other, the iron line disappears exactly at the moment of the reburst. We also point out that a line feature, with a similar significance, has been found by ASCA in another burst, GRB 970828, which also shows an event of rebursting during the X-ray afterglow (Yoshida et al. 1999). On the contrary, neither GRB 971214 nor GRB 98613 show rebursting (Costa 1999).

Acknowledgements. We thank the BeppoSAX team for the support with observations. BeppoSAX is a program of the Italian space agency (ASI) with the participation of the Dutch space agency (NIVR).

\section{References}

Bloom J.S., Djorgovski S.G., Kulkarni S.R., Frail D.A., 1998, ApJL 507, 25

Boella G., et al., 1997, A\&AS 122, 299

Boettcher M., Dermer C.D.D., Crider A.W., Liang E.D., 1999, A\&A 343, 111

Costa E., et al., 1997, Nat 387, 783

Costa E., 1999 (these proceedings)

Djorgovski S.G., et al., 1999, ApJ (in press)

Ghisellini G., et al., 1998, ApJ (submitted; see also these Proc.)

Kulkarni S.R., et al., 1998, Nat 395, 663

Lazzati D., Ghisellini G., Campana S., 1999, MNRAS (in press; see also these Proc.)

Mészáros P., Rees M.J., 1998, MNRAS 299, L10

Metzger M.R., et al., 1997, Nat 387, 878

Perna R., Loeb A., 1998, ApJ 501, 467

Piro L., Scarsi L., Butler R.C., 1995, in: X-Ray and EUV/FUV Spectroscopy and Polarimetry, Fineschi S. (ed.), SPIE 2517, p. $169-181$

Piro L., et al., 1998a, A\&A 329, 906

Piro L., et al., 1998b, A\&A 331, L41

Piro L., et al., 1999, ApJL 514, L73

van Paradijs, et al., 1997, Nat 386, 686

Vietri M., Stella L., 1998, ApJ 507, L45

Yoshida A., et al., 1999 (these proceedings) 